Math. Model. Nat. Phenom.

Vol. 5, No. 7, 2010, pp. 145-150

DOI: $10.1051 / \mathrm{mmnp} / 20105724$

\title{
Spreadability, Vulnerability and Protector Control
}

\author{
A. Bernoussi * \\ Laboratory MPPRN, Faculty of Sciences and Techniques \\ B.P. 416, Tangier, Morocco
}

\begin{abstract}
In this work, we present some concepts recently introduced in the analysis and control of distributed parameter systems: Spreadability, vulnerability and protector control. These concepts permit to describe many biogeographical phenomena, as those of pollution, desertification or epidemics, which are characterized by a spatio-temporal evolution.
\end{abstract}

Key words: distributed systems, spreadability, vulnerability, remediability, protector control AMS subject classification: 37N99, 93A30, 93B99

\section{Introduction}

Some biogeographical phenomena, which are characterized by a spatio-temporal evolution are described by distributed parameter systems. For such systems, the recent works developed by A. El Jai and his team show the importance of spatial variable in the study of these systems. Indeed, in [1], a more appropriate notion to highlight the spatial aspect in the analysis and control was introduced: the regional analysis. That consists in studying the system either on all the space domain $\Omega$ where the state is defined or on a particular subregion $\omega$ (fixed) of $\Omega$. This opened the way to approach distributed systems. More particularly, to describe some environmental phenomena such as pollution, epidemics or fires of forests and so on. The concept of spreadability was introduced by A. El Jai and K. Kassara [7] and developed by A. Bernoussi and A. El Jai [4, 5]. Later the concept of vulnerability was introduced by A. Bernoussi and M. Amharref [6], [3] in order to characterize the zones which can be reached by a given property $\mathcal{P}$ (pollution for example). In addition, L Afifi et al. introduced in [1] the remediability concept. It consists in compensation at the final

*Corresponding author. E-mail: a.bernoussi@uae.ma ; bersamed16@yahoo.fr 
time $T$, by an adequate control, the effect of a known or unknown disturbance $f$ on the state of the system in a given zone $\sigma \subset \Omega$. Recently, protector control was introduced by A. Bernoussi et al for linear systems. In this work, we present the achievement of the development of such concepts. To illustrate our approach, example of transport system is presented.

\section{Spreadability and vulnerability}

Let $(S)$ be a dynamical system defined in $\Omega \subset \mathbb{R}^{n}$ with a state denoted by $z(x, t)=z\left(x, t, t_{0}, z_{0}\right)$ $t \geq t_{0}, \quad x \in \Omega, t_{0}$ is the initial time and $z_{0}(x)=z\left(x, t_{0}\right)$. We assume the following evolution property:

$$
z\left(x, t, t_{0}, z_{0}\right)=z\left(x, t, s, z\left(\cdot, s, t_{0}, z_{0}\right)\right), \quad t_{0} \leq s \leq t .
$$

Let $\omega_{t}$ be the set of all points $x \in \Omega$ such that the state $z(x, t)$ satisfies a given property $\mathcal{P}$ at time $t$

$$
\omega_{t}=\omega_{t}\left(t_{0}, z_{0} ; \mathcal{P}\right)=\left\{x \in \Omega \mid \mathcal{P} z\left(x, t, t_{0}, z_{0}\right)\right\} .
$$

The property $\mathcal{P}$ can be, in the pollution problem for example, the fact that the pollution concentration $(z(x, t))$ is greater than a threshold $C^{*}$ and $\omega_{t}$ is the zone where the pollutant concentration is greater than $C^{*}[2]$. For an epidemic problem, $\omega_{t}$ is the infected zone at time $t[5]$.

Definition 1. - The system $(S)$ is said to be $\mathcal{P}$-spreadable (respectively $\mathcal{A}$-spreadable or spreadable in measure) from $\omega_{t_{0}}$ during the time horizon $I=\left[t_{0}, T\right]\left(T>t_{0}\right)$ if the family of sets $\left(\omega_{t}\right)_{t \in I}$ is increasing (respectively $\mu\left(\omega_{t} \backslash \omega_{s}\right) \geq \mu\left(\omega_{s} \backslash \omega_{t}\right) ; \forall t, s, t_{0} \leq s \leq t \leq T$ where $\mu(\omega)$ is the Lebesgue measure of $\omega$.

- In the particular case where $\mathcal{P}$ is the property that $z(x, t)=0$, the system $(S)$ is said to be null-spreadable.

- We say that a given region $\sigma$ is $\mathcal{P}$-vulnerable if there exist an instant $t \in] t_{0}, T[$ such that: $\stackrel{\circ}{\circ} \omega_{t} \neq \emptyset$. $\stackrel{\circ}{ }$ is the interior of $\sigma$.

To characterize the $\mathcal{A}$-spreadability, A. Bernoussi and A. El Jai [4] have introduced a family of transformations which permit to describe the space evolution of the subdomain $w_{t}=\{x \in \Omega$ : $\mathcal{P} z(x, t)\}$. We recall the following definition [4] in the case of null spreadability.

Definition 2. We say that a family of transformations $(F(., .,)):. \Omega \times \Delta \rightarrow \Omega$ is adapted to the evolution of $(S)$ if there exists a function $\eta: \Omega \times \Delta \rightarrow \mathbb{R}$ such that:

$z[F(x, s, t) ; s]=\eta(x, s, t) z(x, t)$, where $\Delta=\{(s, t) \in I \times I: t \leq s\}$.

Consequently, we have the characterization of spreadability:

Proposition 3. $(S)$ is $\mathcal{A}$-spreadable (respectively $\mathcal{A}$-resorbable) during the time interval I if there exists a family of transformations $(F(., s, t))$ adapted to $(S)$ such that:

$\mu\left(F\left(\omega_{t}, s, t\right) \backslash F\left(\omega_{t}, t, t\right)\right) \geq \mu\left(F\left(\omega_{t}, t, t\right) \backslash F\left(\omega_{t}, s, t\right)\right) ; \forall t, s, t_{0} \leq s \leq t \leq T$. (respectively

$\left.\mu\left(F\left(\omega_{t}, s, t\right) \backslash F\left(\omega_{t}, t, t\right)\right) \leq \mu\left(F\left(\omega_{t}, t, t\right) \backslash F\left(\omega_{t}, s, t\right)\right) ; \forall t, s, t_{0} \leq s \leq t \leq T.\right)$ 
A moving disturbance can be, during a time interval, $\mathcal{A}$-spreadable or $\mathcal{A}$-resorbable or can alternate between being $\mathcal{A}$-spreadable and $\mathcal{A}$-resorbable. In both cases it can reach $\sigma$. We have the following characterization:

Proposition 4. (i) A zone $\sigma$ is vulnerable if there exists a family of mappings $(F(., s, t))$ adapted to the evolution of $(S)$ and $t_{1} \in I$ such that: $\stackrel{\circ}{\sigma} \cap F\left(\omega_{t_{0}}, t_{1}, t_{0}\right) \neq \emptyset$.

(ii) Denote $\tau_{T}=\cup_{s \in] t_{0}, T[} F\left(\omega_{0}, s, t_{0}\right)$ : the trajectory of the property $\mathcal{P}$, then $\sigma$ is vulnerable if and only if there exists a time $t_{1} \in I$ such that: $\stackrel{\circ}{\sigma} \cap \tau_{t_{1}} \neq \emptyset$ ( $\sigma$ is in the trajectory of $\left.\mathcal{P}\right)$.

For more details about spreadability see $[4,5,8,9]$ and vulnerability $[3,6]$. An application to the groundwater pollution is given in [2].

\section{Protector control}

In this section, we consider the problem of protection of a given vulnerable zone face to a moving phenomenon. For example for cancer spreadability, when we cannot remedy it, we try to protect the vital organs. As another application in flood or fire of forest problems, when we cannot stop the phenomenon, we try to protect in priority the more interesting areas: habitations or industrial installations. To describe such problems, we consider a linear system $(S)$ which is disturbed by a disturbance $f$ and controlled by $u$ :

$$
(S)\left\{\begin{array}{l}
\dot{z}(t)=A z(t)+G f(t)+B u(t) \quad 0<t<T, \\
z(0)=z_{0},
\end{array}\right.
$$

where $A$ generates a strongly continuous semi group $(S(t))_{t \geq 0}$ on the state space $X=L^{2}(\Omega), \Omega$ is an open and bounded subset of $\mathbb{R}^{n}, B \in \mathcal{L}\left(\mathcal{U} ; L^{2}(\Omega)\right), u \in L^{2}(0, T ; \mathcal{U}), \mathcal{U}$ is an Hilbert space, $f \in L^{2}(0, T ; \mathcal{F})$ is a disturbance and $G \in \mathcal{L}\left(\mathcal{F} ; L^{2}(\Omega)\right) . X, \mathcal{U}$ and $\mathcal{F}$ denote respectively the state, control and disturbance spaces. Let $\sigma$ be a given (fixed) region of $\Omega$. Assume that the system $(S)$ is augmented by the measure function given by: $y^{\sigma}(t)=C i_{\sigma} p_{\sigma} z(t)$ where $C \in \mathcal{L}\left(L^{2}(\Omega), Y\right)$, $Y$ is an Hilbert space (observation space), $p_{\sigma}$ is the restriction to $\sigma$ and $i_{\sigma}$ its adjoint operator. The solution of (3.1) is given by:

$$
z_{u, f}(t)=S(t) z_{0}+H_{t} u+\widetilde{H}_{t} f
$$

where

$$
H_{t} u=\int_{0}^{t} S(t-s) B u(s) d s \text { and } \widetilde{H}_{t} f=\int_{0}^{t} S(t-s) G f(s) d s
$$

and then

$$
y_{u, f}^{\sigma}(t)=C i_{\sigma} p_{\sigma} S(t) z_{0}+C i_{\sigma} p_{\sigma} H_{t} u+C i_{\sigma} p_{\sigma} \widetilde{H}_{t} f .
$$

The remediability concept was introduced by Afifi et al. [1]. The principle consists, in the regional case, in compensating by an adequate control, the disturbance on the state of the system $(S)$ in the zone $\sigma$ at the final time $T$. This problem is formulated as follow:

$$
(P)\left\{\begin{array}{l}
\text { Does a control } u \in L^{2}(0, T ; \mathcal{U}) \text { such that : } \\
C i_{\sigma} p_{\sigma} H_{T} u+C i_{\sigma} p_{\sigma} \widetilde{H}_{T} f=0
\end{array}\right.
$$


We have the following definition [1]:

Definition 5. 1 . The system $(S)$ is said to be exactly $f$ - $\sigma$-remediable (or $f$ - $\sigma$-remediable) during $[0, T]$ if there exists a control $u \in L^{2}(0, T ; \mathcal{U})$ such that: $C i_{\sigma} p_{\sigma} H_{T} u+C i_{\sigma} p_{\sigma} \widetilde{H}_{T} f=0$.

2. $(S)$ is said to be weakly $f$ - $\sigma$-remediable during $[0, T]$ if for all $\varepsilon>0$, there exists a control $u \in L^{2}(0, T ; \mathcal{U})$ such that: $\left\|C i_{\sigma} p_{\sigma} H_{T} u+C i_{\sigma} p_{\sigma} \widetilde{H}_{T} f\right\| \leq \varepsilon$.

Using some classical results on controllability, we have the following result [1]:

Proposition 6. 1. The system $(S)$ is exactly $f$ - $\sigma$-remediable during $[0, T]$ if and only if: $C i_{\sigma} p_{\sigma} \widetilde{H}_{T} f \in \operatorname{Im}\left(C i_{\sigma} p_{\sigma} H_{T}\right)$.

2. The system $(S)$ is weakly $f$ - $\sigma$-remediable during $[0, T]$ if and only if $C i_{\sigma} p_{\sigma} \widetilde{H}_{T} f \in \overline{\operatorname{Im}\left(C i_{\sigma} p_{\sigma} H_{T}\right)}$.

In some situations, as for an incurable epidemic (Aids or cancer for example), it is impossible to remedy a given zone if it was reached or the remediable control exists but it is at very high cost (pollution). Face to such situations, we have considered the following problem: Is it possible to protect such a zone before that it will be reached? This is the principle of protector control. The protector control problem is formulated as follow:

$$
(P 1)\left\{\begin{array}{l}
\text { Determinate a control } u \in L^{2}(0, T ; \mathcal{U}) \text { such that : } \\
\left.C i_{\sigma} p_{\sigma} H_{t} u+C i_{\sigma} p_{\sigma} \widetilde{H}_{t} f=0 ; \forall t \in\right] 0, T[.
\end{array}\right.
$$

Starting from the above formulation of the problem $(P 1)$, we introduce the concept of protector control. In this sense, we have the following definition.

Definition 7. 1. The zone $\sigma$ is said to be f-protectable if:

$$
\exists u \in L^{2}(0, T ; \mathcal{U}): C i_{\sigma} p_{\sigma} H_{t} u+C i_{\sigma} p_{\sigma} \widetilde{H}_{t} f=0 \quad \forall t \in[0, T] .
$$

In this case $u$ is said to be $f$ - $\sigma$-protector control.

2. $\sigma$ is said to be weakly $f$-protectable if:

$$
\forall \varepsilon>0, \exists u \in L^{2}(0, T ; \mathcal{U}):\left\|C i_{\sigma} p_{\sigma} H_{t} u+C i_{\sigma} p_{\sigma} \widetilde{H}_{t} f\right\|_{Y} \leq \varepsilon \quad \forall t \in[0, T] .
$$

In this case we say that $u$ is a weakly $f$ - $\sigma$-protector control.

A $f$ - $\sigma$-protector control is $f$ - $\sigma$-remediable. The converse is not true. We have the characterization of such a control using an adaptable family of transformations .

Proposition 8. A control $u$ is a protector one if and only if it is associated to an adaptable family of transformation $\left(F(., s, t)\right.$ ) (the family is adapted to the system excited by $u$ ) satisfying $\tau_{T} \cap \stackrel{\circ}{\sigma}=$ $\left(\bigcup_{t \in I} F\left(w_{u, f, 0}, t, 0\right)\right) \cap \stackrel{\circ}{\sigma}=\emptyset$.

To determinate a protector control for a given vulnerable zone $\sigma$, we process in two steeps. First

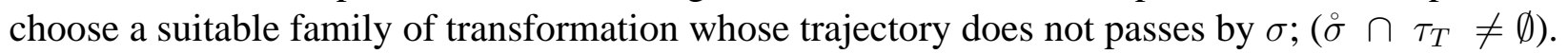
Second, find a control $u$ associated to the chosen family of transformations. 


\section{Example: Transport system}

To illustrate our approach, consider the following transport system given by:

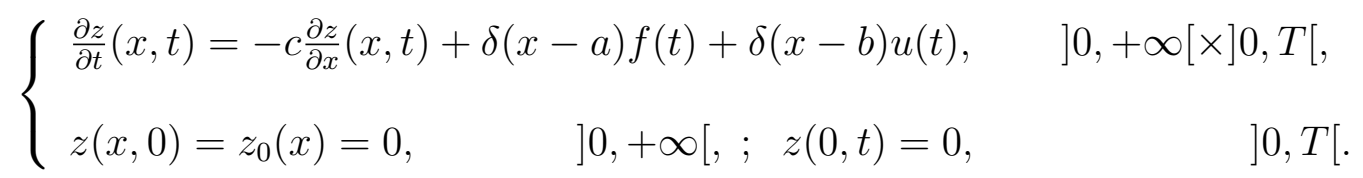

Where $a, b, c>0, \delta$ is the Dirac function and $f$ is a disturbance. We assume that $T \leq \frac{a}{c}$, then in the autonomous case $(u=0)$, the solution is given by:

$$
z_{0, f}(x, t)=\left\{\begin{array}{lr}
f\left(\frac{a-x}{c}+t\right), & a \leq x \leq a+c t \\
0, & \text { elswhere }
\end{array}\right.
$$

Denote $w_{0, f, t}=\left\{x \in \Omega: z_{0, f}(x, t) \neq 0\right\}$, then $w_{0, f, t}=[a, a+c t]$. The system (disturbance) is spreadable and for all $\sigma=[\alpha, \beta]$ fixed in $\Omega$, If $\beta<a$ then $\sigma$ is non vulnerable but $\sigma$ is vulnerable if $\beta>a$. By a suitable control (protector control) we can make $\sigma$ non vulnerable. For example consider the case where $a=2, \alpha=3, \beta=4, T=8 ; c=1 / 4$. In the autonomous case $\sigma$ is $f$-vulnerable but the pointwise control located at $b=3$ and given by $u(t)=-f(t-4)$ is a $\sigma$-protector control.

\section{Conclusion}

In this paper, we have presented some new concepts introduced in order to describe some biogeographical phenomena. The new points of view to consider distributed systems have been appeared since the introduction of the regional analysis notion by A. El Jai. The spreadability and vulnerability describe many biogeographical phenomena and protector control permits to protect some vulnerable area when we can not stop the disturbance spreadability. It will be very interesting to extend this results to nonlinear and chaotic systems in the aim to describe a more realistic phenomena.

\section{Acknowledgements}

This work is supported by the Académie Hassan II des Sciences et Techniques.

\section{References}

[1] L. Afifi, A. Chafiai, A. El Jai. Regionally efficient and strategic actuators. International Journal of Systems Science, 33 (2002), 1-12. 
[2] M. Amharref, S. Aassine, A. Bernoussi, B. Haddouchi. Carthographie de la vulnérabilité à la pollution des eaux souterraines: Application à la plaine du Gharb. Revue des Sciences de l'Eau, 20 (2007), No. 2 , 185-199.

[3] A. Bernoussi. Spreadability and vulnerability of distributed parameter systems. International Journal of System Sciences, 38 (2007), No. 4, 305-317.

[4] A. Bernoussi, A. El Jai. New approach of spreadability. Journal of Mathematical and Computer Modelling, (2000), No. 31, 93-109.

[5] A. Bernoussi, A. El Jai, A. J. Pritchard. Spreadability and evolving interfaces. International Journal of Systems Science, 32 (2001), No. 10, 1217-1232.

[6] A. Bernoussi, M. Amharref. Etalabilité - vulnérabilité. Annals of University of Craiova, Math. Comp. Sci. Ser., 30 (2003), 53-62.

[7] A. El Jai, K. Kassara. Spreadable distributed systems. Mathematical and Computer Modelling, 20 (1994), No. 1, 47-64.

[8] A. El Jai, K. Kassara. Spreadability of transport systems. International Journal of Systems Science, 27 (1996), No. 7, 681-688.

[9] D. Ucinski, A. El Jai. On weak spreadability of DPS and its achievement via LQ control techniques. IMA Journal on Control and Information Science, 14 (1997), 153-164. 